\title{
Seasonal Variations of Polarization Diversity Gain in a Vegetated Area considering High Elevation Angles and a Nomadic User
}

\author{
Milan Kvicera and Pavel Pechac \\ Department of Electromagnetic Field, Faculty of Electrical Engineering, Czech Technical University in Prague, Technicka 2, \\ 16627 Prague 6, Czech Republic \\ Correspondence should be addressed to Milan Kvicera; kvicemil@fel.cvut.cz
}

Received 20 September 2014; Revised 1 December 2014; Accepted 8 December 2014

Academic Editor: Jit S. Mandeep

Copyright ( 2015 M. Kvicera and P. Pechac. This is an open access article distributed under the Creative Commons Attribution License, which permits unrestricted use, distribution, and reproduction in any medium, provided the original work is properly cited.

\begin{abstract}
Seasonal variations of the polarization diversity gain are addressed for a nomadic user in a vegetated area taking high elevation angles and nongeostationary satellites into consideration. Corresponding experimental data were obtained at a frequency of $2.0 \mathrm{GHz}$ at Stromovka Park in Prague, the Czech Republic, within the full in-leaf and out-of-leaf periods of 2013 and 2014, respectively. By detecting copolarized and cross-polarized components of the transmitted left- and right-handed circularly polarized signals, the corresponding diversity gain was obtained for multiple-input single-output (MISO), single-input multiple-output (SIMO), and combined MISO/SIMO cases. It was found that tree defoliation results in a significant decrease of the polarization diversity gain achieved for low time percentages in particular scenarios.
\end{abstract}

\section{Introduction}

One of the standard satellite-to-earth scenarios of the future is a nomadic user in an outdoor vegetated area relying on satellite services in a variety of locations over an extended period of time. Such a user can be characterized as being static for the time spent within a particular location. As satellites on nongeostationary Earth orbits, such as medium or low Earth orbits, provide the required high-data rates with small time delays, the position of a particular satellite, with respect to the user, may change dramatically within the period of time the user remains in a fixed position, in contrast to low elevation links such as in [1].

Mobile $[2,3]$ or static [4-6] users have been the focus of most studies in vegetated areas. On the one hand, in order to characterize a propagation channel for a static user, a representative scenario is selected and a vast range of elevation and azimuth angles is considered to account for different satellite positions. On the other hand, the land mobile propagation channel may be characterized by static elevation and azimuth angles while focusing on rapidly changing user surroundings.
Characterizing the propagation channel for a nomadic user is more complex. Apart from specifying a scenario of interest, one must take into account the wide range of elevation and azimuth angles achieved between the user and a nongeostationary Earth orbit satellite for arbitrarily fixed positions of the user terminal within the scenario. Consequently, there are an unlimited number of users, surroundings, and satellite orientations, each of which impacts upon the corresponding propagation channel [7]. Thus, from a measurement campaign point of view, it is feasible to address typical representative scenarios only.

It should be noted that, apart from the empirical approach presented in this paper, attenuation and scattering by vegetation can also be treated in an analytical way as, for example, in the case of the multiple scattering theory [8] where lossy dielectric cylinders and thin disks are utilized to model a tree canopy within which their distribution is considered to be uniform $[9,10]$ or more specific [11].

Considering a vegetated area at different seasons, which have a significant impact from the propagation point of view $[4,12]$, full in-leaf and out-of-leaf conditions can be achieved. 
As polarization diversity at the satellite's transmitter and multiple antennas at the user terminal are expected to ensure a certain quality of service [13], it is of great interest to investigate the corresponding diversity gain with respect to its seasonal variations. As tree defoliation has mainly been addressed from the vegetation attenuation point of view for the case of a single-input single-output propagation channel $[4,12]$, its influence on the polarization diversity gain has not been thoroughly documented in the literature.

Thus, experimental data regarding the influence of tree defoliation on the polarization diversity gain are not publicly available, apart from [3] where the nomadic satellite channel is considered, albeit only for the case of a geostationary satellite and full in-leaf vegetation. This is why we have performed a series of measurements at $2.0 \mathrm{GHz}$ in a vegetated area in Prague, the Czech Republic. During these trials, a remotecontrolled airship was utilized as a pseudosatellite following predefined fly paths. Transmit (Tx) antennas were instantly pointed towards a receiver $(\mathrm{Rx})$ kept in a fixed position. These trials were performed at four selected representative scenarios and, to achieve both summer and winter seasons' measurements, they were carried out in July, 2013, and March, 2014, respectively. As both the left-handed (LHCP) and right-handed (RHCP) circularly polarized continuous wave signals were transmitted at the same time and a dualpolarized receive antenna was utilized, seasonal variations of the diversity gain for the case of multiple-input single-output (MISO), single-input multiple-output (SIMO), and combined MISO/SIMO (represented here by two inputs and two outputs) propagation channels were addressed. Contrasted to $[4,5]$ or $[6]$, in addition to the Tx antenna positioner, such measurements were made possible by utilizing a sensitive, custom-made $\mathrm{Rx}$ with four channels sampled in parallel at a rate of $10 \mathrm{kHz}$.

Section 2 describes the measurement setup and selected scenarios while Section 3 introduces the data processing method. Section 4 presents the obtained results and their analysis. Section 5 discusses the influence of the $\mathrm{Rx}$ antenna radiation patterns on the experimental data and Section 6 concludes this paper.

\section{Measurement Setup and Selected Scenarios}

The measurement setup includes an LHCP and an RHCP transmit planar wideband spiral antenna attached at the bottom of the airship to a 3D positioner enabling instant pointing towards the location of the $\mathrm{Rx}$ based on the airship GPS coordinates. Two unmodulated continuous wave signals separated by a $200 \mathrm{kHz}$ frequency offset were transmitted with a stable, constant output power of $27 \mathrm{dBm}$ and were received by a dual-circularly-polarized (LHCP and RHCP) antenna. This antenna consisted of a dual-linearly-polarized patch antenna and an H-hybrid coupler which shifted the received orthogonal linearly polarized components by $90^{\circ}$ in phase in order to create RHCP and LHCP signals; see Figure 1 for more details. The $\mathrm{Rx}$ antenna was placed on a tripod at a height of 1.5 meters above ground level and oriented so that its main lobe pointed towards the zenith. Received

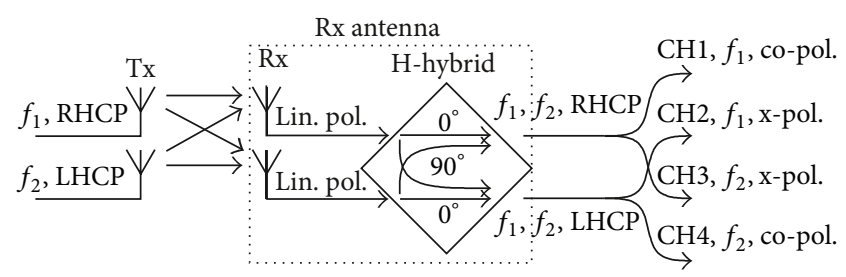

FIGURE 1: The measurement system in detail. One RHCP and one LHCP continuous wave signals at frequencies $f_{1}$ and $f_{2}$, respectively, are transmitted and their orthogonal components with a linear polarization are received by a dual-linearly-polarized patch antenna. An H-hybrid coupler then creates RHCP and LHCP signals at its output ports so that the copolarized (co-pol.) and cross-polarized (x-pol.) components of the transmitted signals can be detected by the four-channel receiver $(\mathrm{CH} 1-\mathrm{CH} 4)$.

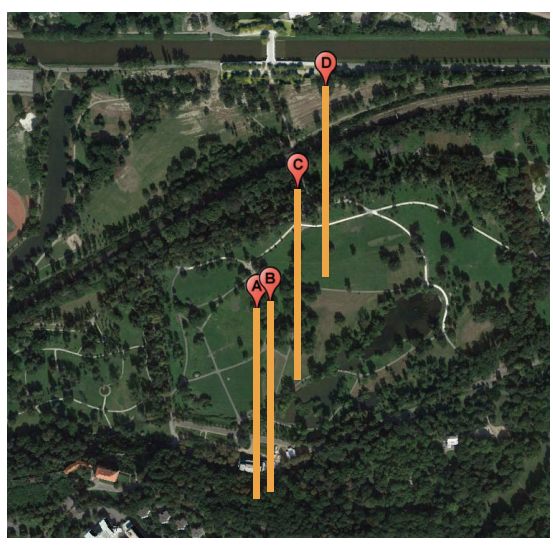

Figure 2: Scenarios A, B, C, and D together with the fly path of the airship denoted by the straight line. The symbols represent the actual positions of the receiver. Image taken from Google Earth.

signal levels were recorded by a computer connected to a sensitive, four-channel radio receiver with a sampling rate of $10 \mathrm{kHz}$ so that four samples were obtained concurrently every $0.1 \mathrm{~ms}$. This custom-made receiver had a low noise floor of $-126 \mathrm{dBm}$ for a $12.5 \mathrm{kHz}$ measurement bandwidth and its first two channels were tuned to $2.00106 \mathrm{GHz}$ and the other two to $2.00086 \mathrm{GHz}$. Both copolarized and cross-polarized components of the transmitted signals were detected enabling MISO/SIMO channel investigations.

Under line-of-sight propagation conditions, the dynamic range of the system is about $45 \mathrm{~dB}$ for the lowest elevation angle achieved, namely, 30 degrees, and about $70 \mathrm{~dB}$ considering the overhead position of the Tx. This considers the free space loss when the altitude of the airship is approximately 200 meters above ground level. Thus, with respect to the roughly $10 \mathrm{~dB}$ vegetation attenuation at $2 \mathrm{GHz}$ for the whole range of elevation angles, as reported in [4], it was possible to detect signal envelope fades of at least $35 \mathrm{~dB}$.

At Stromovka Park, the following scenarios for a nomadic user in a vegetated area were selected as shown in Figures 2 and 3. Within scenarios $\mathrm{A}$ and $\mathrm{B}$, the $\mathrm{Rx}$ location was next to and inside an alley of deciduous trees. Scenario $C$ represented a heavily shadowed case with the $\mathrm{Rx}$ in the middle of a dense 

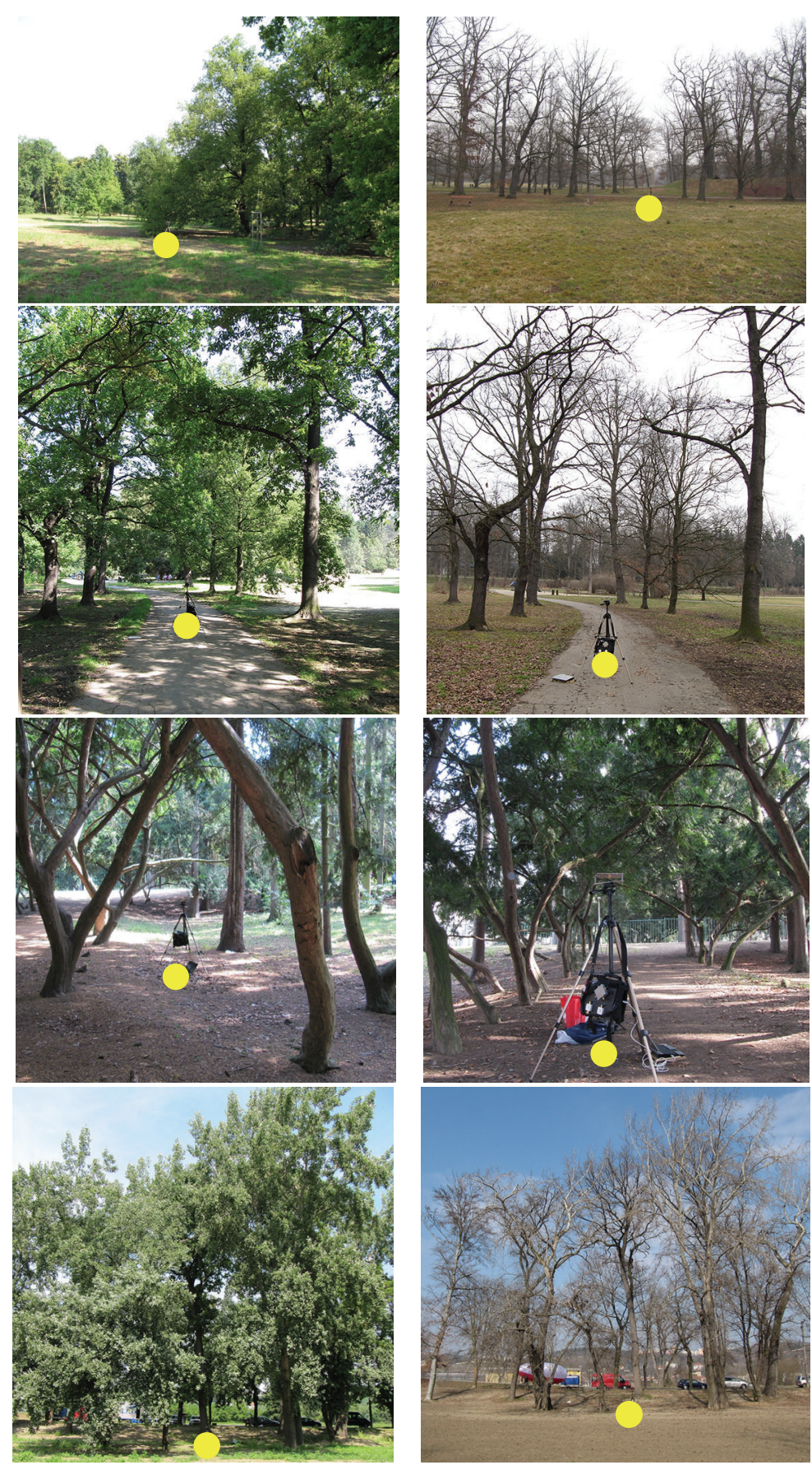

Figure 3: From top to bottom: scenarios A, B, C, and D in detail during the summer (on the left) and winter (on the right) seasons together with the $\mathrm{Rx}$ position denoted by a circle.

group of coniferous trees, while at scenario $\mathrm{D}$, the $\mathrm{Rx}$ was surrounded by a group of tall deciduous trees. In this way, a representative set of scenarios was addressed enabling a generalization of the obtained results.

The airship followed a predefined, straight flyover above these scenarios in a south-north direction with a near-constant speed of about $8 \mathrm{~m} / \mathrm{s}$, as indicated in Figure 2, allowing a wide range of elevation angles starting from 30 degrees to be achieved. Moreover, the flyovers were almost perfectly aligned with the orientation of the alley for scenarios $\mathrm{A}$ and B. During gusty conditions, the transmit antenna positioner did not keep the direction towards the Rx perfectly and, thus, the data obtained within airship pitch and roll periods of more than 15 degrees were excluded from data processing. Further, similar to [4], the influence of free space loss for various distances between the Tx and the Rx was removed by recalculating received signal levels to a uniform distance of $20 \mathrm{~km}$. It should be noted that measurement time stamps were 
used to synchronize the experimental data with the flight data provided by the airship's sensors.

\section{Data Processing}

As the experimental data contain copolarized and crosspolarized components of the transmitted signals $P_{\mathrm{TxRx}}$ in $\mathrm{dBm}$, they were processed according to (5)-(10) by using the maximum ratio combining (MRC) approach $[2,14]$ to calculate the corresponding diversity gain $G$ for the MISO, SIMO, and combined MISO/SIMO cases.

First, the amplitude of the received power $p_{\mathrm{TxRx}}$ in linear units had to be obtained for each of the four received channels (1) based on the levels $P_{\text {TxRx }}$ which were recorded in $\mathrm{dB}$ :

$$
p_{\mathrm{TxRx}}=10^{P_{\mathrm{TXR}} / 10} .
$$

Here, the first and second indices Tx and Rx stand for the polarization of the transmitted and received signals, represented in the following text by indices $\mathrm{R}$ and $\mathrm{L}$ denoting RHCP and LHCP, respectively.

Then, the maximum ratio combining algorithm was applied and an equivalent envelope power $p_{\text {eq }}$ was calculated. As [14] states that the signal-to-noise ratio (SNR) is simply the sum of the SNRs of the individual signal branches for the MRC algorithm, the SIMO case considering a RHCP transmitted signal can be written as

$$
\begin{gathered}
\mathrm{SNR}_{\mathrm{SIMO}}=\frac{p_{\mathrm{RR}}}{N}+\frac{p_{\mathrm{RL}}}{N}=\frac{p_{\mathrm{eq}, \mathrm{SIMO}}}{N}, \\
p_{\mathrm{eq}, \mathrm{SIMO}}=p_{\mathrm{RR}}+p_{\mathrm{RL}},
\end{gathered}
$$

where an uncorrelated noise of power $N$ is assumed to be received by both signal branches. To obtain the polarization diversity gain in $\mathrm{dB}$, the equivalent envelope power is expressed in logarithmic scale and the copolarized signal power $P_{\mathrm{RR}}$ is subtracted:

$$
G_{\mathrm{SIMO}, \mathrm{RHCP}}=10 \cdot \log _{10}\left(p_{\mathrm{RR}}+p_{\mathrm{RL}}\right)-P_{\mathrm{RR}} \cdot
$$

As there were two separate transmitters with an equal output power for the MISO and MISO/SIMO cases during the experimental trials, the combined powers must be divided by 2 so that the total output power is the same as that for the SIMO case. As a result, (5)-(7) and (8)-(10) were applied for the RHCP and LHCP cases, respectively:

$$
\begin{gathered}
G_{\mathrm{MISO}, \mathrm{RHCP}}=10 \cdot \log _{10}\left[\frac{\left(p_{\mathrm{RR}}+p_{\mathrm{LR}}\right)}{2}\right]-P_{\mathrm{RR}}, \\
G_{\mathrm{SIMO}, \mathrm{RHCP}}=10 \cdot \log _{10}\left(p_{\mathrm{RR}}+p_{\mathrm{RL}}\right)-P_{\mathrm{RR}},
\end{gathered}
$$

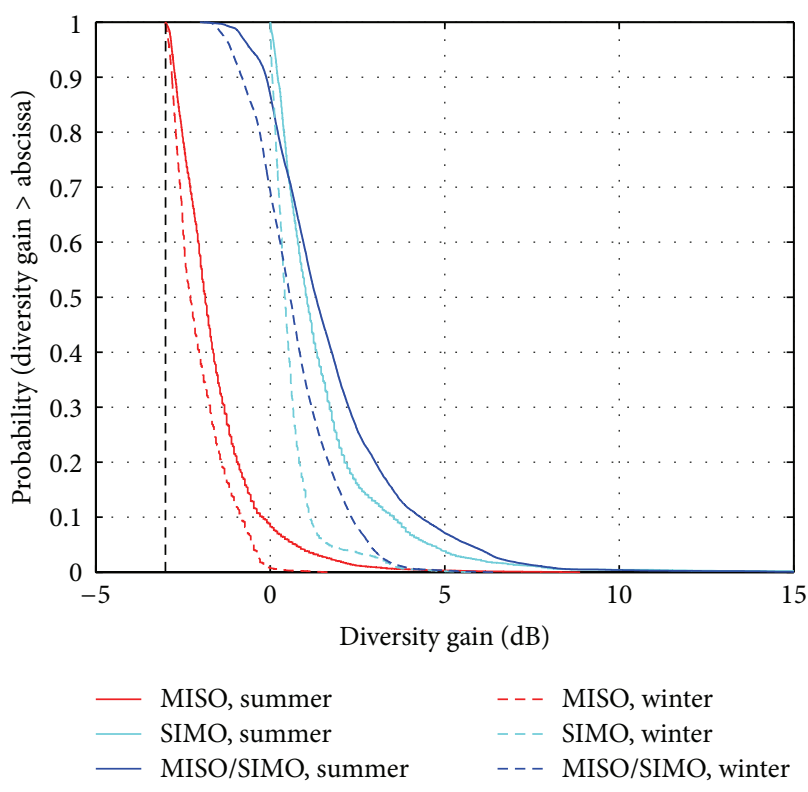

FIGURE 4: CCDF of the diversity gain for scenario $\mathrm{B}, 30^{\circ}-40^{\circ}$.

$$
\begin{aligned}
& G_{\mathrm{MISO} / \mathrm{SIMO}, \mathrm{RHCP}} \\
& =10 \cdot \log _{10}\left[\frac{\left(p_{\mathrm{RR}}+p_{\mathrm{RL}}+p_{\mathrm{LR}}+p_{\mathrm{LL}}\right)}{2}\right]-P_{\mathrm{RR}}, \\
& G_{\mathrm{MISO}, \mathrm{LHCP}}=10 \cdot \log _{10}\left[\frac{\left(p_{\mathrm{LL}}+p_{\mathrm{RL}}\right)}{2}\right]-P_{\mathrm{LL}}, \\
& G_{\mathrm{SIMO}, \mathrm{LHCP}}=10 \cdot \log _{10}\left(p_{\mathrm{LL}}+p_{\mathrm{LR}}\right)-P_{\mathrm{LL}}, \\
& G_{\mathrm{MISO} / \mathrm{SIMO}, \mathrm{LHCP}} \\
& =10 \cdot \log _{10}\left[\frac{\left(p_{\mathrm{RR}}+p_{\mathrm{RL}}+p_{\mathrm{LR}}+p_{\mathrm{LL}}\right)}{2}\right]-P_{\mathrm{LL}} .
\end{aligned}
$$

\section{Results and Analysis}

Prior to providing an overall analysis for the whole range of elevation angles, it was necessary to address the seasonal variations of the polarization diversity gain within a range of elevation angles where the large-scale variations of the received signal envelope may be neglected. For such purposes, ten-degree intervals in elevation were selected as the propagation conditions, together with copolarized and crosspolarized radiation patterns of the Rx antenna, do not change considerably within this range and, moreover, a sufficient amount of data samples are available to ensure their thorough statistical interpretation.

The polarization diversity gain achieved at scenario B during the summer and winter seasons is presented in Figures $4-6$ for the intervals at elevations of $30^{\circ}-40^{\circ}, 50^{\circ}-60^{\circ}$, and $70^{\circ}-80^{\circ}$. This scenario represents heavy tree shadowing propagation conditions and since it is leafless during the winter season, corresponding seasonal variations of the polarization diversity gain should be the most significant from all of 

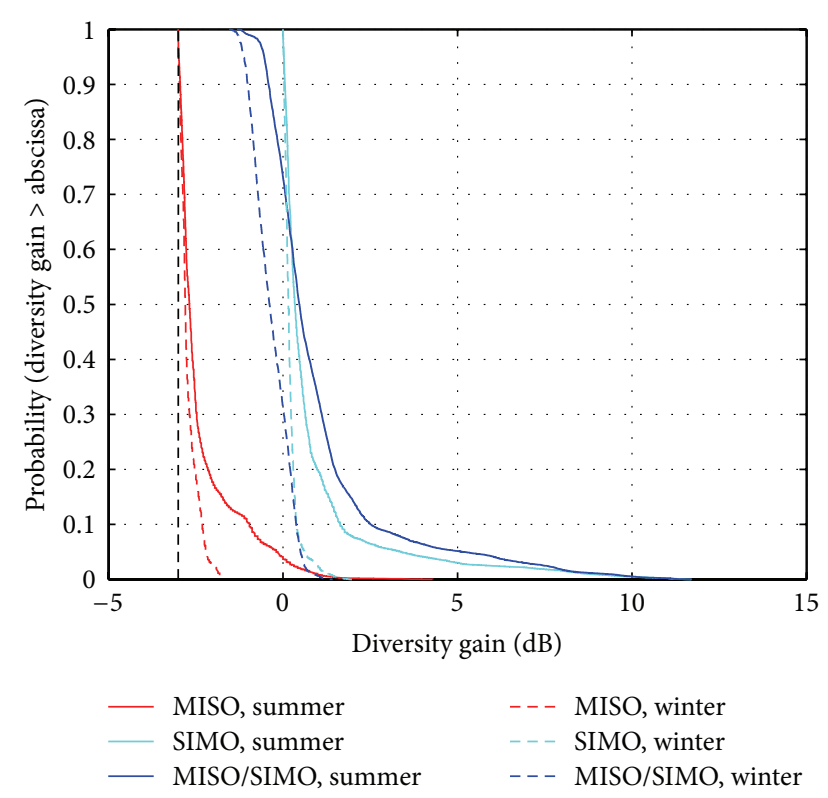

FIGURE 5: CCDF of the diversity gain for scenario $\mathrm{B}, 50^{\circ}-60^{\circ}$.

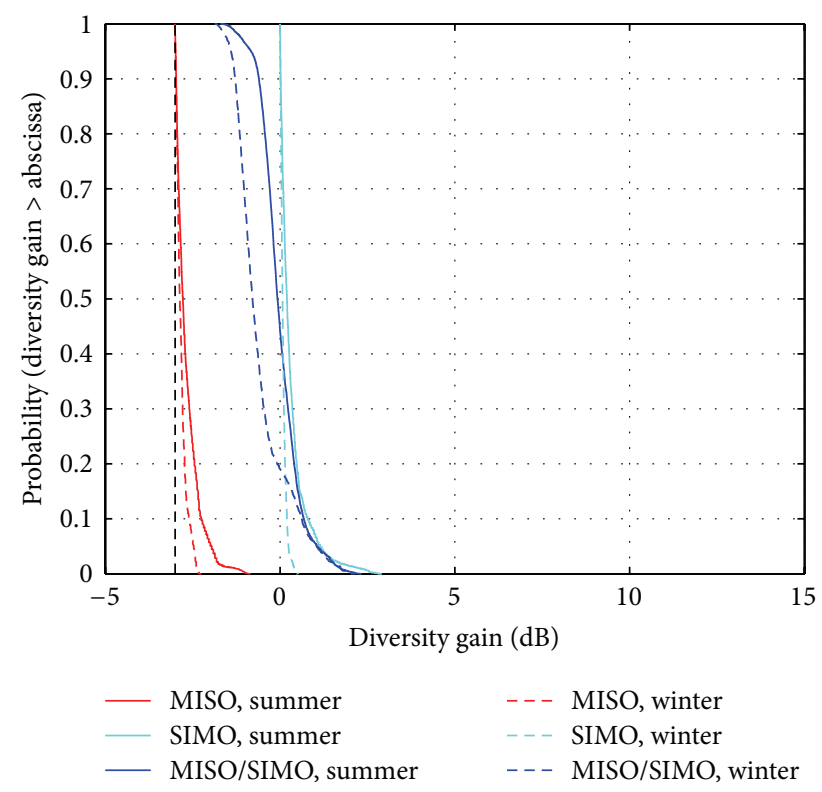

Figure 6: CCDF of the diversity gain for scenario $\mathrm{B}, 70^{\circ}-80^{\circ}$.

the addressed scenarios. Complementary cumulative distribution functions (CCDFs) of the polarization diversity gain, defined as

$$
\operatorname{Pr}\left\{G>G_{\text {th }}\right\}=1-\int_{-\infty}^{G_{\text {th }}} p(G) \mathrm{d} G
$$

calculated using the MATLAB ecdf function, clearly demonstrate, as seen in Figures 4-6, a significant decrease within the winter season, especially for low time percentages below $10 \%$. It should be noted that the MISO case generally loses $3 \mathrm{~dB}$ of the diversity gain due to the consideration of two transmitters; see (2). The $-3 \mathrm{~dB}$ border lines are, for reasons

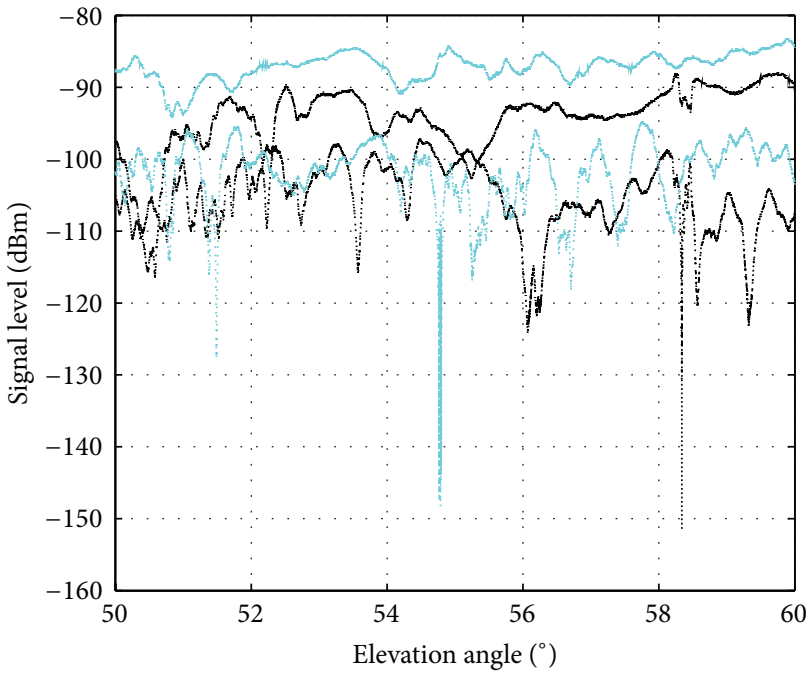

$\begin{array}{llll} & \mathrm{RR} \text {, summer } & & \mathrm{RR} \text {, winter } \\ \ldots \ldots & \mathrm{RL} \text {, summer } & \mathrm{RL} \text {, winter }\end{array}$

FIgURE 7: Time series of received signal levels for scenario B recalculated to a uniform distance of $20 \mathrm{~km}$, elevation $50^{\circ}-60^{\circ}$. The first and second indices in the legend represent the polarization of the transmitted and received signals with $\mathrm{R}$ and $\mathrm{L}$ representing RHCP and LHCP, respectively.

of clarity, denoted in Figures 4-6 by a separate dashed vertical line.

Considering, for example, the elevation interval of $50^{\circ}$ to $60^{\circ}$ (Figure 5), a more detailed analysis can be presented based on the raw time series of the copolarized and crosspolarized received signal levels and their CCDFs; see Figures 7 and 8 , respectively. A median $6 \mathrm{~dB}$ decrease of the copolarized received signal level for the summer season is obvious in Figure 8 due to the additional attenuation caused by the presence of leaves. This decrease can also be observed in the raw time series data shown in Figure 7. On the other hand, apart from significant small-scale variations of the cross-polarized components clearly seen in Figure 7, their median decrease is only $3 \mathrm{~dB}$ for the summer season as seen in Figure 8 . This indicates that, in accordance with [12], the presence of branches is more significant than the presence of leaves when the scattering phenomenon is considered. Similar behavior was observed for all ten-degree elevation intervals from 30 to 80 degrees at all the investigated scenarios, making it possible to proceed with the analysis and provide results for the whole range of elevation angles, as shown in Figures 9-12.

To compare the polarization diversity gain achieved at the selected scenarios during the summer and winter seasons, corresponding CCDFs are shown in Figures 9-12. Here, the range of elevation angles between 30 degrees and 80 degrees was considered as it contained the majority of the experimental data. Producing a CCDF of the diversity gain for such a wide range of elevation angles takes into account the fast movement of the satellites on nongeostationary Earth orbits during the time period the user is in a fixed position. 


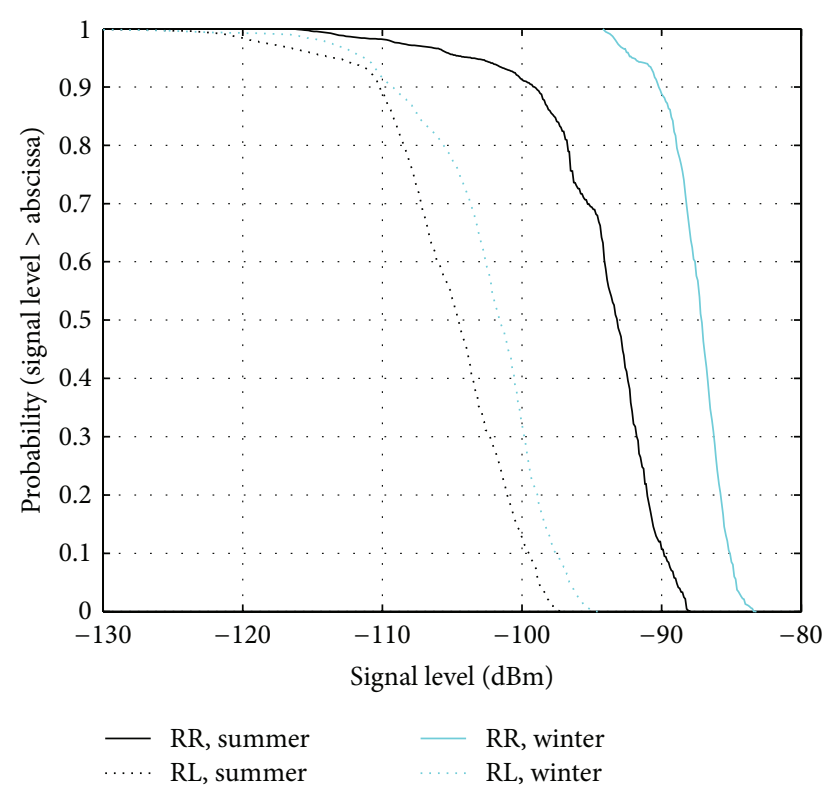

FIGURE 8: CDF of received signal levels for scenario $\mathrm{B}$ recalculated to a uniform distance of $20 \mathrm{~km}$, elevation $50^{\circ}-60^{\circ}$. The first and second indices in the legend represent the polarization of the transmitted and received signals with $\mathrm{R}$ and $\mathrm{L}$ representing RHCP and LHCP, respectively.

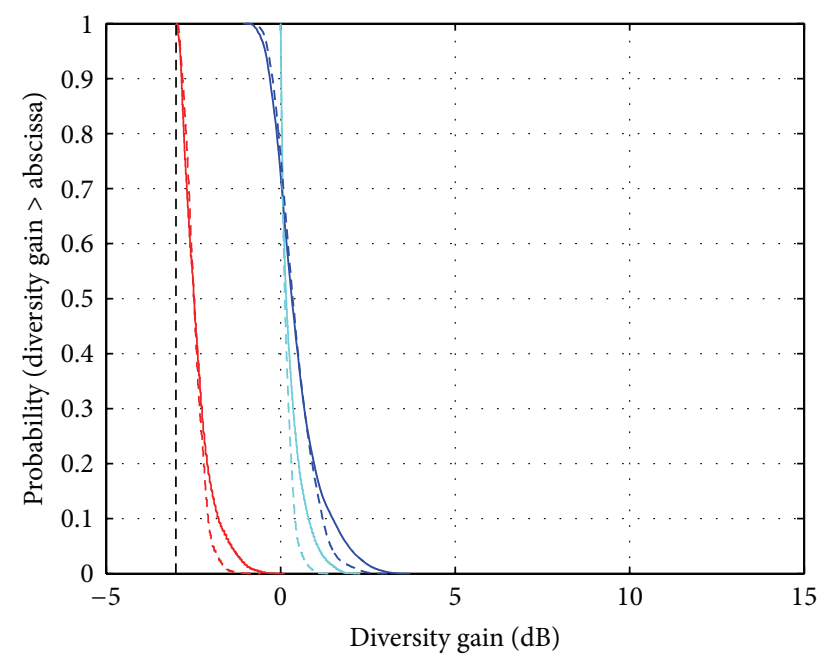

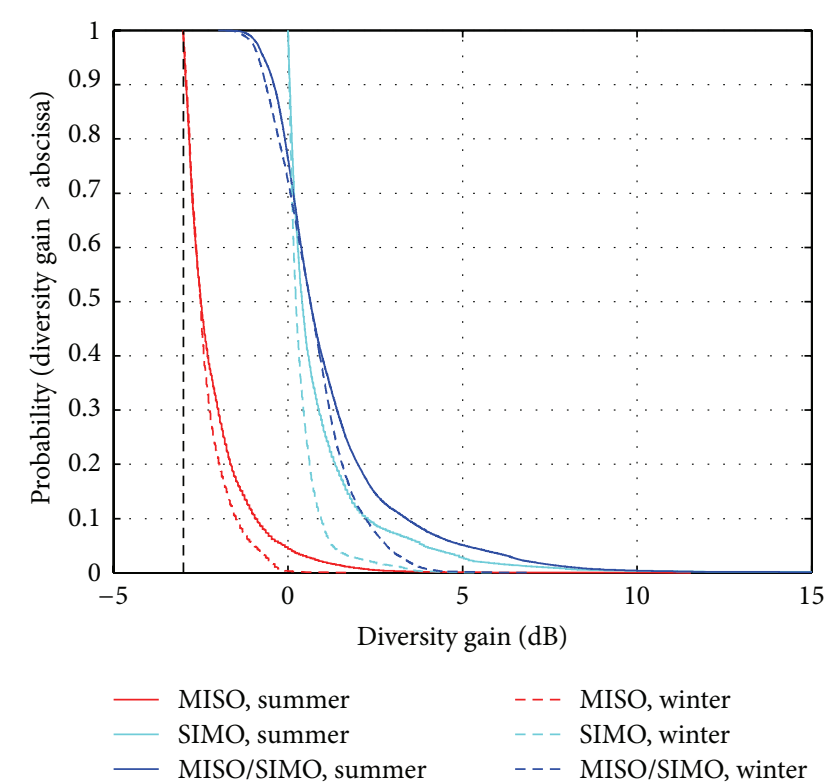

FIgURE 10: CCDF of the diversity gain for scenario B.

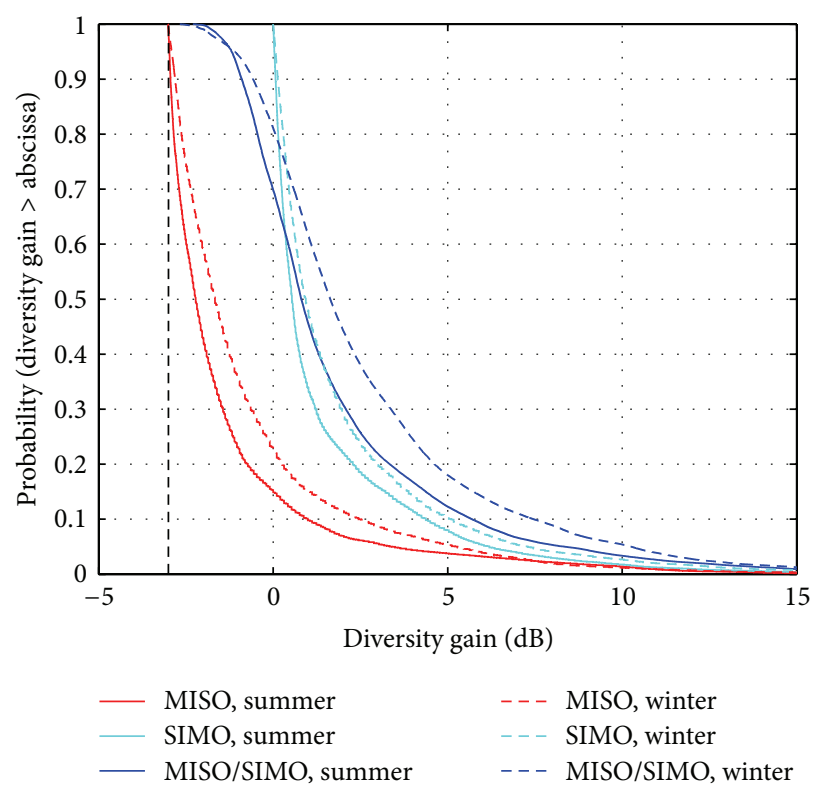

FIGURE 11: CCDF of the diversity gain for scenario C.

the mean scattering amplitude per unit volume of the tree canopy depends linearly on the density of branches or leaves. Thus, for more vegetated scenarios with rich scattering, more scattered power should be received resulting in an increase of the diversity gain.

Apart from such a brief analysis, the polarization diversity gain differences for the summer and winter seasons are addressed below. Figure 9 shows that even though the trees in the alley are leafless during the winter season a negligible decrease of only about $0.5 \mathrm{~dB}$ of the diversity gain for time percentages below $10 \%$ can be observed when the $\mathrm{Rx}$ was

located next to the alley. The effect of the leafless season
As the results obtained for the LHCP and RHCP cases were similar, only the RHCP case is shown in Figures 9-12.

It is clear that when considering time probabilities below $50 \%$, the highest and lowest diversity gains were achieved at scenario $\mathrm{C}$, the most vegetated, and at scenario $\mathrm{A}$, with the $\mathrm{Rx}$ located next to an alley, respectively. As expected, the more vegetated the scenario, the more significant the benefits of the diversity approach. This can be explained by [12] where 

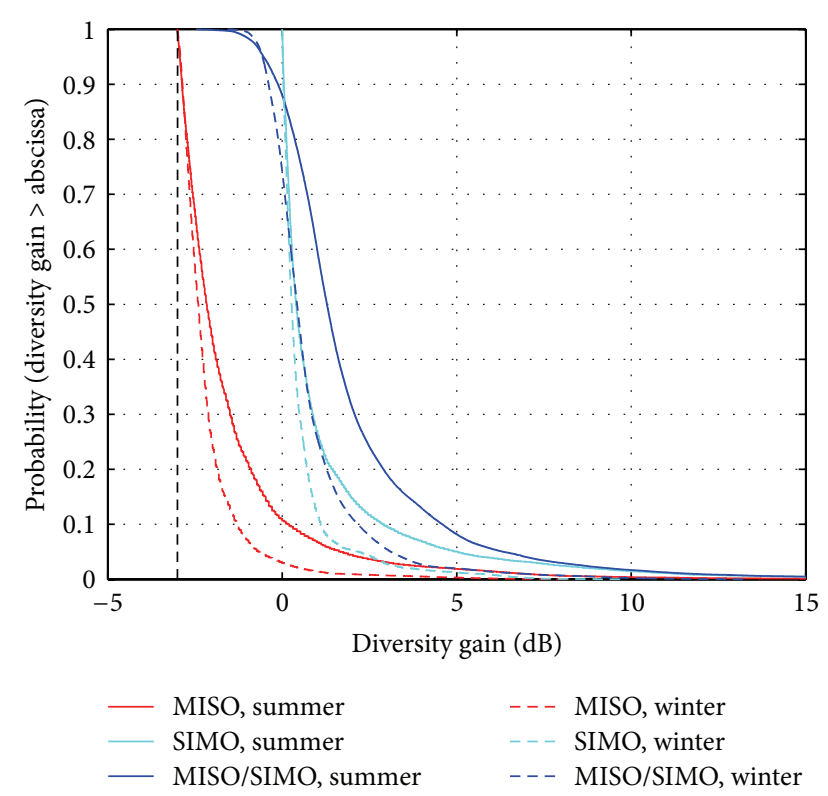

FIGURE 12: CCDF of the diversity gain for scenario D.

is clearly limited by the existence of almost clear line-ofsight propagation conditions on one side of the Rx. On the other hand, more than a $2 \mathrm{~dB}$ decrease of the diversity gain for the winter season is observed in Figure 5 for scenario $\mathrm{B}$ when considering time percentages below $10 \%$, which demonstrates the significant effect of defoliated trees. As scenario $\mathrm{C}$ comprises only evergreen coniferous trees, one would expect the diversity gain to be the same during both the summer and winter seasons. However, following the airship flyover denoted in Figure 2, the $\mathrm{Rx}$ was located deeper in the vegetated area during the winter season measurements due to maintenance works in the park area. This resulted in about a $1 \mathrm{~dB}$ higher diversity gain illustrating the advantage of diversity systems for more shadowed scenarios.

Results similar to those obtained at scenario B were identified for scenario $\mathrm{D}$ which also consists of deciduous trees. However, these trees do not form an alley and, based on Figure 3, the effect of defoliation was expected to be more pronounced here. This is shown well in Figure 7 where, for time percentages of $10 \%$, the diversity gain decreases from approximately $4.6 \mathrm{~dB}$ in the summer season to about $2.2 \mathrm{~dB}$ in the winter season considering the MISO/SIMO case.

\section{Influence of Rx Antenna Radiation Pattern}

Considering the results presented in Figures 4-6 and 9-12, it is important to address their dependence on the fact that, although the copolarized radiation patterns of the $\mathrm{Rx}$ antenna are similar, the cross-polarized radiation patterns differ by up to $5 \mathrm{~dB}$ for elevation angles below 50 degrees according to the simulations performed in CST Microwave Studio using the time domain solver. This is illustrated in Figure 13 which presents the dependence of the $\mathrm{Rx}$ antenna gain on elevation angle when considering a phi-cut of $90^{\circ}$ and theta angles from $-60^{\circ}$ to $0^{\circ}$ which translate into elevation angles from $30^{\circ}$

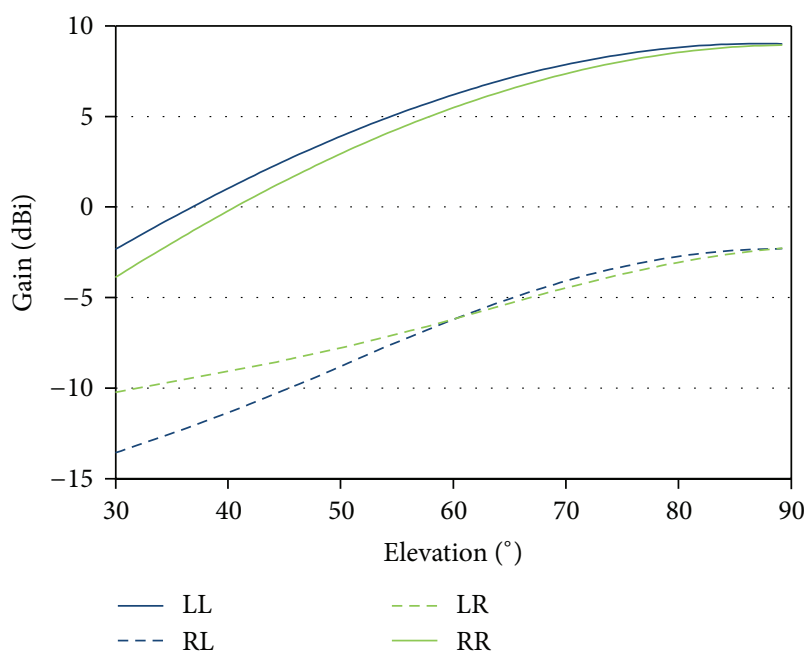

FIGURE 13: Dependence of the $\mathrm{Rx}$ antenna gain on elevation angle considering the copolarized (LL, RR) and cross-polarized (RL, LR) radiation patterns.

to $90^{\circ}$. This feature of a dual-polarized antenna, together with the inability to compensate for the radiation pattern in an environment where the scattered waves are impinging on the Rx from a wide range of elevation and azimuth angles, results in the need to address the error introduced by such radiation patterns asymmetries.

Following (3) for the SIMO case, it is straightforward to calculate the difference in the diversity gain $\Delta$ in $\mathrm{dB}$ when the level of the received cross-polarized component is changed by a dimensionless parameter $\alpha$. This parameter represents a deviation from an ideal radiation pattern, that is, the one which would be the same for both the cross-polarized RHCP and LHCP cases, and we can write

$$
\begin{aligned}
\Delta & =G_{\text {SIMO,RHCP actual }}-G_{\text {SIMO,RHCP ideal }} \\
& =10 \cdot \log \left(1+\alpha \frac{p_{\mathrm{RL}}}{p_{\mathrm{RR}}}\right)-10 \cdot \log \left(1+\frac{p_{\mathrm{RL}}}{p_{\mathrm{RR}}}\right) .
\end{aligned}
$$

Using $\chi$ to represent the ratio between the cross-polarized and copolarized received signal levels $p_{\mathrm{RR}}$ and $p_{\mathrm{RR}}$, (12) reduces to

$$
\Delta=10 \cdot \log \left(\frac{1+\alpha \chi}{1+\chi}\right)
$$

where

$$
\chi=10 \cdot \exp \left(0.1 \cdot\left(P_{\mathrm{RL}}-P_{\mathrm{RR}}\right)\right)
$$

considering that $P_{\mathrm{RL}}$ and $P_{\mathrm{RR}}$ are the received signal levels in $\mathrm{dBm}$. Further, as $A$ defines the difference from the ideal crosspolarized radiation pattern in $\mathrm{dB}$, we can write

$$
\alpha=10 \cdot \exp (0.1 \cdot A)
$$

Based on the Microwave CST simulations of the $\mathrm{Rx}$ antenna, a typical value of a $10 \mathrm{~dB}$ difference between $P_{\mathrm{RL}}$ and $P_{\mathrm{RR}}$ and the maximum value of $A$ of about $5 \mathrm{~dB}$ may be 
considered. Then, one obtains $\Delta$ of $0.78 \mathrm{~dB}$ and $-0.28 \mathrm{~dB}$ for cases where $A=5 \mathrm{~dB}$ and $A=-5 \mathrm{~dB}$, respectively, which plays a negligible role in the presented results, especially when considering the low time percentages below $10 \%$.

It should be noted that the expressions for the MISO case reduce after basic logarithm calculations to the form of (13), where $\chi$ represents the ratio between $p_{\mathrm{LR}}$ and $p_{\mathrm{RR}}$. For the combined MISO/SIMO case, (13) can be used if $P_{\mathrm{RR}}=P_{\mathrm{LL}}$ and $P_{\mathrm{LR}}=P_{\mathrm{RL}}$ are considered.

\section{Conclusion}

We have presented a thorough analysis of polarization diversity gain measurements at $2.0 \mathrm{GHz}$ for a nomadic user in a vegetated area considering high elevation angles and both the summer and winter seasons. The case of orthogonal circular polarizations was considered. It was shown that, depending on the scenario, tree defoliation has a significant impact on the achieved diversity gain regardless of the MISO, SIMO, or combined MISO/SIMO configuration. At first, this was demonstrated on experimental data selected within tendegree intervals in elevation and, after that, for the whole range of elevation angles from 30 degrees to 80 degrees. In addition, the influence of asymmetrical cross-polarized radiation patterns of the $\mathrm{Rx}$ antenna on the diversity gain calculations was addressed. Based on a typical discrimination of the copolarized and cross-polarized radiation patterns of the Rx antenna, only a negligible impact on the presented results was found.

Considering time percentages below $10 \%$, we have shown that tree defoliation resulted in a negligible decrease of $0.5 \mathrm{~dB}$ of the diversity gain when the Rx was located next to the alley, but a decrease of more than $2 \mathrm{~dB}$ was observed for the case of the Rx located inside the alley. Similar results were obtained when the $\mathrm{Rx}$ was in the middle of a group of deciduous trees. Here, the diversity gain decreased from about $4.6 \mathrm{~dB}$ in the summer season to about $2.2 \mathrm{~dB}$ in the winter season for the combined MISO/SIMO case. Considering the scenario consisting of evergreen coniferous trees, the $\mathrm{Rx}$ was located deeper in the vegetated area during the winter season measurements, which resulted in an increase of about $1 \mathrm{~dB}$ of the diversity gain.

As a rule of thumb, we have demonstrated that the polarization diversity gain achieved in the full in-leaf season decreases in the leafless season to half of its value when considering time percentages below 50\% for the SIMO and combined MISO/SIMO case for the case of the maximum ratio combining algorithm. This rule can be utilized to estimate polarization diversity gain seasonal variations and can thus decrease the complexity of corresponding measurement campaigns which would have to be carried out during both the full in-leaf and out-of-leaf conditions.

\section{Conflict of Interests}

The authors declare that there is no conflict of interests regarding the publication of this paper.

\section{Acknowledgment}

This work was supported by the European Social Fund within the framework of realizing the project "Support of InterSectoral Mobility and Quality Enhancement of Research Teams at Czech Technical University in Prague" (CZ.1.07/ 2.3.00/30.0034).

\section{References}

[1] M. Simunek, P. Pechac, and F. P. Fontan, "Feasibility of UAV link space diversity in wooded areas," International Journal of Antennas and Propagation, vol. 2013, Article ID 890629, 5 pages, 2013.

[2] F. Lacoste, F. Carvalho, F. Perez-Fontan, A. Nunez-Fernandez, V. Fabro, and G. Scot, "MISO and SIMO measurements of the land mobile satellite propagation channel at S-band," in Proceedings of the 4th European Conference on Antennas and Propagation (EuCAP '10), pp. 1-5, Barcelona, Spain, April 2010.

[3] F. Lacoste, J. Lemorton, L. Casadebaig, and F. Rousseau, "Measurements of the land mobile and nomadic satellite channels at $2.2 \mathrm{GHz}$ and $3.8 \mathrm{GHz}$," in Proceedings of the 6th European Conference on Antennas and Propagation (EuCAP '12), pp. 2422-2426, Prague, Czech Republic, March 2012.

[4] P. Horak and P. Pechac, "Seasonal additional attenuation in woodlands for satellite services at L-, S- and C-bands," IEEE Transactions on Antennas and Propagation, vol. 59, no. 12, pp. 4865-4867, 2011.

[5] C. I. Kourogiorgas, M. Kvicera, D. Skraparlis et al., "Modeling of first-order statistics of LMS channel under tree shadowing for various elevation angles at L-band," in Proceedings of the 8th European Conference on Antennas and Propagation (EuCAP '14), pp. 2273-2277, The Hague, The Netherlands, April 2014.

[6] C. Kourogiorgas, M. Kvicera, D. Skraparlis et al., "Modeling of first-order statistics of the MIMO dual polarized channel at $2 \mathrm{GHz}$ for land mobile satellite systems under tree shadowing," IEEE Transactions on Antennas and Propagation, vol. 62, no. 10, pp. 5410-5415, 2014.

[7] M. Rieche, A. Ihlow, D. Arndt, F. Pérez-Fontán, and G. Del Galdo, "Modeling of the land mobile satellite channel considering the terminal's driving direction," International Journal of Antennas and Propagation, vol. 2014, Article ID 372124, 35 pages, 2014.

[8] V. Twersky, "Multiple scattering of electromagnetic waves by arbitrary configurations," Journal of Mathematical Physics, vol. 8, no. 3, pp. 589-610, 1967.

[9] M. A. Karam, A. K. Fung, and Y. M. Antar, "Electromagnetic wave scattering from some vegetation samples," IEEE Transactions on Geoscience and Remote Sensing, vol. 26, no. 6, pp. 799808, 1988.

[10] S. A. Torrico and R. H. Lang, "A simplified analytical model to predict the specific attenuation of a tree canopy," IEEE Transactions on Vehicular Technology, vol. 56, no. 2, pp. 696703, 2007.

[11] Y.-C. Lin and K. Sarabandi, "A Monte Carlo coherent scattering model for forest canopies using fractal-generated trees," IEEE Transactions on Geoscience and Remote Sensing, vol. 37, no. 1, pp. 440-451, 1999.

[12] Y. L. C. de Jong and M. H. A. J. Herben, "A tree-scattering model for improved propagation prediction in urban microcells," IEEE Transactions on Vehicular Technology, vol. 53, no. 2, pp. 503-513, 2004 . 
[13] P.-D. Arapoglou, K. Liolis, M. Bertinelli, A. Panagopoulos, P. Cottis, and R. De Gaudenzi, "MIMO over satellite: a review," IEEE Communications Surveys and Tutorials, vol. 13, no. 1, pp. 27-51, 2011.

[14] S. R. Saunders and A. Aragón-Zavala, Antennas and Propagation for Wireless Communication Systems, 2nd edition, 2007. 

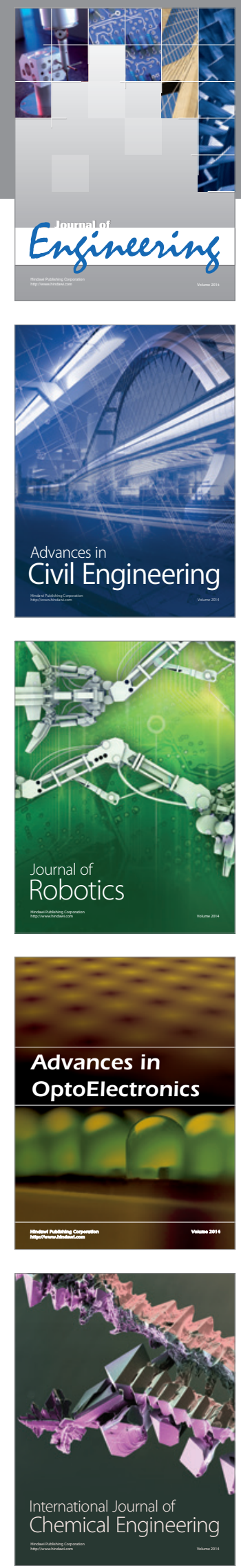

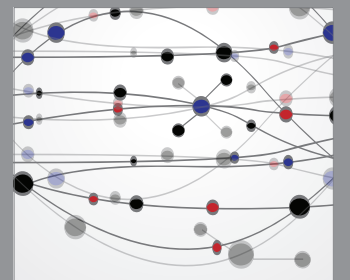

The Scientific World Journal
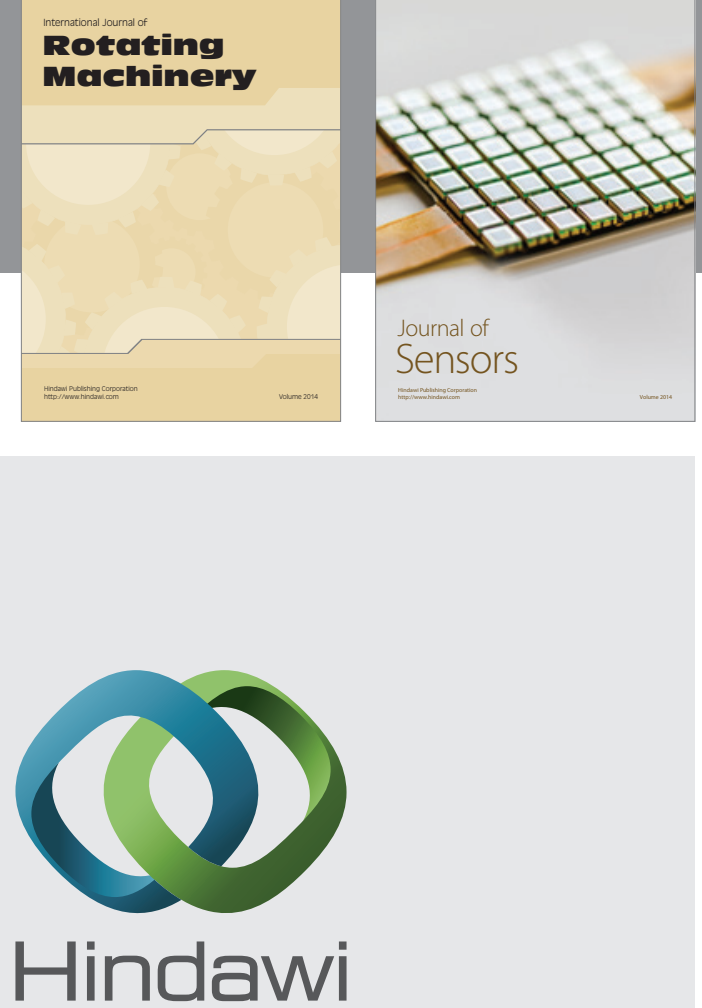

Submit your manuscripts at http://www.hindawi.com
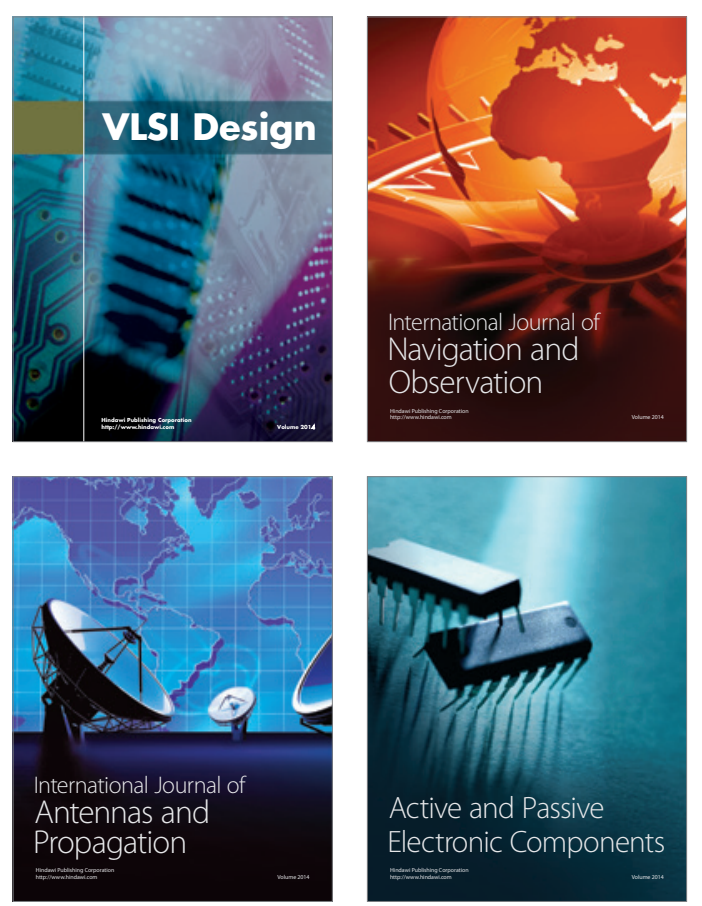
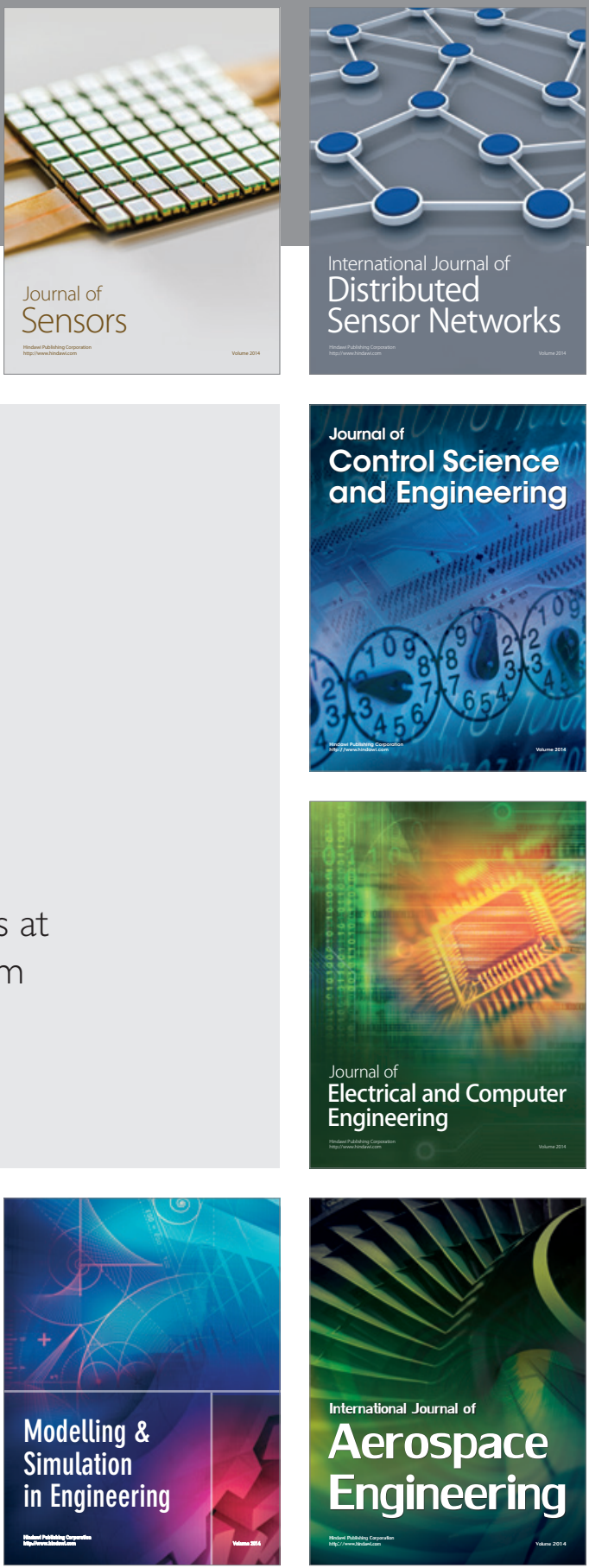

Journal of

Control Science

and Engineering
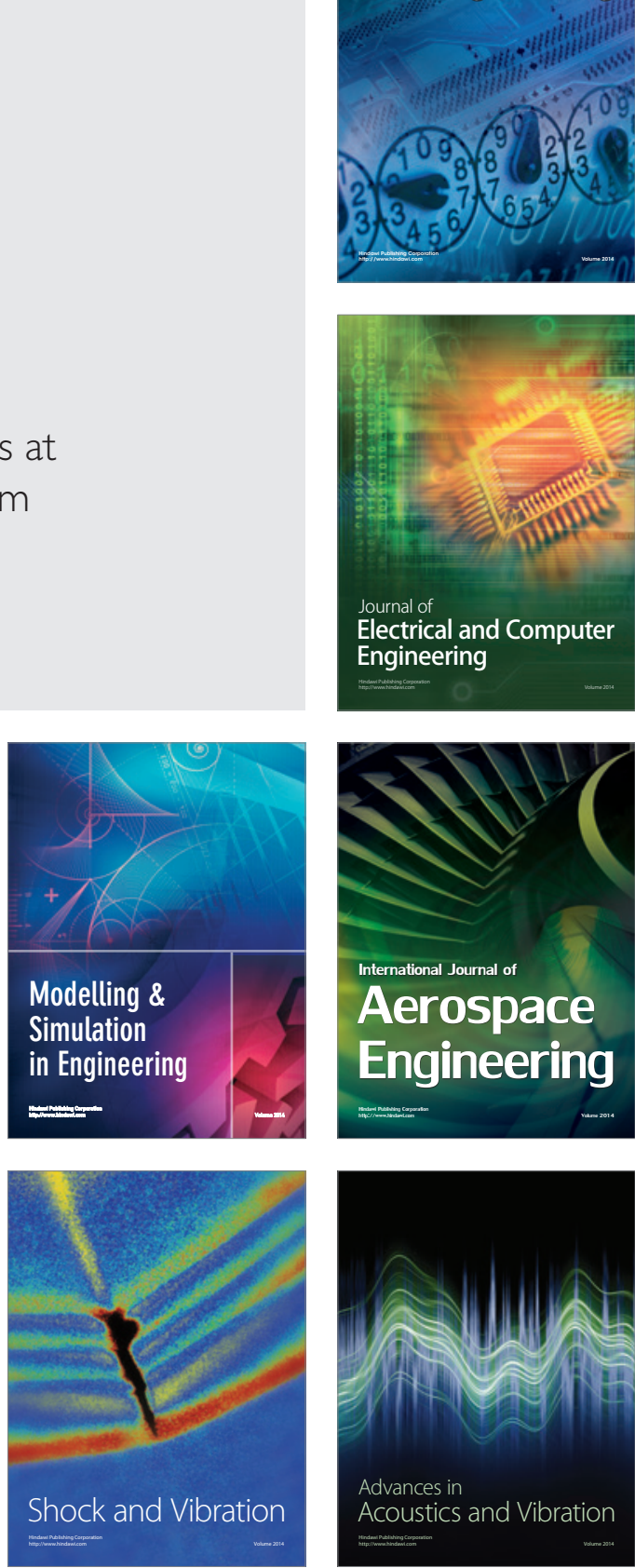